\title{
Nationwide survey of US integrated 6-year cardiothoracic surgical residents
}

\author{
Amir H. Lebastchi, MD, and David D. Yuh, MD
}

\begin{abstract}
Objective: Integrated 6-year cardiothoracic surgical residency programs have recently been implemented in the United States. We report the results of the first published nationwide survey assessing the motivations, satisfaction, and ambitions of integrated 6-year residents.
\end{abstract}

Methods: A 63-question web-based survey was distributed to 83 residents enrolled in 21 Accreditation Council for Graduate Medical Education-accredited integrated 6-year programs in November 2013. There was an outstanding $69 \%$ response rate.

Results: The median age of integrated 6-year residents was 29 years with women comprising $24 \%$. A clear majority had faculty mentorship (95\%) and significant clinical exposure in medical school. Focused (100\%) and abbreviated (74\%) training curricula were identified as the top advantages of integrated 6-year programs; the format itself was a significant factor $(46 \%)$ in career choice. Most integrated 6-year residents $(95 \%)$ were satisfied with their program; $80.7 \%$ were satisfied with their operative experience thus far. Career plans skewed toward adult cardiac surgery $(67 \%)$, followed by pediatric cardiac $(24 \%)$ and general thoracic $(9 \%)$ surgery; $49 \%$ were not particularly concerned about future employment, with $65 \%$ foreseeing an increase in opportunities. Specialized training (eg, aortic, heart failure, minimally invasive, congenital) was anticipated by $77 \%$. Most integrated 6-year residents envision an academic career $(94.7 \%)$.

Conclusions: This survey takes an important snapshot of the nascent integrated 6-year format. Mentorship and intense clinical exposure are critical in attracting applicants. Purported advantages of the format are holding true among integrated 6-year residents, with the majority satisfied with their programs. These early data indicate that this format holds significant promise in attracting and retaining highly qualified trainees to academic cardiothoracic surgery. (J Thorac Cardiovasc Surg 2014;148:401-7)

The past decade has witnessed a decline in the number of qualified applicants to US cardiothoracic surgical residencies, resulting in a surplus of unfilled training positions and closure of some programs. ${ }^{1-5}$ This decline has occurred despite significant technologic advances in the field, a growing proportion of older patients with cardiovascular disease, ${ }^{6}$ and an accelerated rate of retirement among experienced cardiac surgeons. ${ }^{7}$ Some of these factors contributed to the conclusions of a Society of Thoracic Surgeons/American Association for Thoracic Surgery-commissioned Association of American Medical Colleges study that projected the demand for cardiothoracic surgeons could increase by as much as $46 \%$ by the year $2025 .{ }^{6}$ The trend toward a reduced number of residency programs and residents in training combined with an increased demand for experienced cardiothoracic surgeons

\footnotetext{
From the Section of Cardiac Surgery, Department of Surgery, Yale University School of Medicine, New Haven, Conn

Disclosures: Authors have nothing to disclose with regard to commercial support.

Received for publication Dec 19, 2013; revisions received March 10, 2014; accepted

for publication March 21, 2014; available ahead of print April 17, 2014.

Address for reprints: David D. Yuh, MD, Section of Cardiac Surgery, Yale University

School of Medicine, 333 Cedar St, Boardman 204, PO Box 208039, New Haven,

CT 06520 (E-mail: david.yuh@yale.edu).

$0022-5223 / \$ 36.00$

Copyright (c) 2014 by The American Association for Thoracic Surgery

http://dx.doi.org/10.1016/j.jtcvs.2014.03.040
}

portends significant, perhaps critical, shortages in the future.

In response to the decline in applicants to cardiothoracic surgical residencies, the advent of multidisciplinary approaches for the treatment of cardiovascular and pulmonary disease, and perhaps inspiration from other highly competitive surgical subspecialties, prominent educators partnered with the American Board of Thoracic Surgeons and Thoracic Surgery Residency Review Committee to redesign the cardiothoracic surgical curriculum. ${ }^{8}$ Emphasis was placed on integrating formalized rotational experiences in medical and surgical subspecialties directly relevant to modern cardiothoracic surgical practice (eg, interventional cardiology/radiology, heart failure, critical care, cardiac anesthesia, vascular surgery) with traditional rotations in adult cardiac, congenital cardiac, general thoracic, and general surgery. In acknowledgment of the disadvantages of the 7- to 10-year duration of traditional cardiothoracic surgical training pathways, this new "integrated" curriculum was focused into a 6-year format.

Because the first integrated 6-year (I-6) program adopted at Stanford University in 2007 has recently graduated their first residents and the number of I-6 programs has steadily increased since its implementation, we thought it both timely and important to better understand the motivations, 


\section{Abbreviations and Acronyms \\ ACGME $=$ Accreditation Council for Graduate Medical Education \\ I-6 = integrated 6-year \\ PGY = postgraduate year}

satisfaction, and ambitions of I-6 residents. We therefore conducted a nationwide survey of I-6 residents in all US Accreditation Council for Graduate Medical Education (ACGME)-accredited I-6 programs and report our results and interpretations.

\section{METHODS}

A web-based electronic survey platform (Qualtrics, Provo, Utah) was distributed to all 83 residents enrolled in 21 ACGME-accredited I-6 cardiothoracic surgical training programs on November 1, 2013; the survey was closed on December 1, 2013 (Table 1). Four newly accredited I-6 programs had not yet enrolled their first residents at the time of this survey and were not included. A $\$ 50$ generic gift-card incentive for completed surveys was offered, and 2 reminder electronic mails for initial nonrespondents were sent. The anonymity of all respondents was preserved. Survey data were exported from the platform in a pure text file format (.CSV) and subjected to basic statistical analysis. An audit of the data revealed neither duplicate responses nor multiple responses from any one individual.

The survey was composed of 63 questions specifically designed to define the demographics, background/prior experience, motivations, satisfaction, and future career plans of current I-6 residents. Of these questions, 32 were multiple-choice with 3 permitting the selection of more than 1 choice and 25 questions were based on a Likert response scale. One question had fields for specific numeric responses. Two questions permitted free text responses. The Yale University Institutional Review Board approved the conduct of this study.

Only data from completed surveys were analyzed. GraphPad Prism software, version 6.0c (GraphPad Software, Inc, La Jolla, Calif) was used for basic statistical analysis and plotting of the data. Responses were computed and presented as frequencies. Free-text responses were qualitatively interpreted and categorized. Because there was no true control group, results were analyzed and presented in a descriptive manner.

\section{RESULTS}

\section{Respondents' Demographics}

In November 2013, there were 21 ACGME-accredited I-6 cardiothoracic surgical residency programs with 1 to 12 residents each. A $69 \%$ response rate was achieved, with 57 residents from 19 programs completing the survey (Table 1). Of the respondents, $13(23 \%)$ were women and $44(77 \%)$ were men with a median age of 29 years. The representation of each of the 6 training years was as follows: postgraduate year (PGY)1 (37\%), PGY2 (19\%), PGY3 (21\%), PGY4 (14\%), PGY5 (5\%), and PGY6 (4\%).

Of those surveyed, $89 \%$ concomitantly applied to both general surgery and I-6 residency programs. Some of the respondents earned additional degrees during their education: $\mathrm{PhD}$ (9\%), MPH (4\%), and MSc (14\%).
Many residents actively engaged in research before entering I-6 training $(72 \%)$; most of their research was based in cardiovascular disease $(68 \%)$.

\section{Exposure to Cardiothoracic Surgery Before Matriculation}

Of the 57 respondents, $93 \%$ rotated onto a cardiothoracic surgery clerkship during medical school with more than $63 \%$ spending 3 or more months on the rotation (Table 2). Approximately half observed (53\%) or actively participated in $(47 \%)$ more than 50 cases.

\section{Career and Integrated Training Motivation}

Previous clinical exposure, an interest in cardiovascular medicine, and mentorship were the most important factors in pursuing a career in cardiothoracic surgery for the majority of respondents $(98 \%, 96 \%$, and $89 \%$, respectively) (Table 2). Of the factors measured, financial compensation was the least important consideration in entering the field, with $43.9 \%$ indicating that it was not important in their decision; salary level perceptions appeared to be slightly below published estimates. ${ }^{9}$ Furthermore, $60 \%$ of respondents were not motivated by earlier earning potential in applying to I-6 programs.

Of note, the I- 6 format itself was a significant draw toward the field in $46 \%$ of the respondents. All of the respondents indicated that the focused curriculum of the I-6 format was the most attractive feature. Other desirable features of the I-6 training pathway included exposure to cardiovascular medicine $(82 \%)$ and pulmonary medicine $(60 \%)$, and the reduced general surgical rotational requirements $(81 \%)$.

\section{Satisfaction With and Prevalence of I-6 Program Curricular Elements}

I-6 residents were "satisfied" or "very satisfied" with their cardiothoracic training (95\%) (Table 3). In fact, if given a second opportunity to choose a residency program, $93 \%$ would again select an I-6 program; 2 respondents indicated that they would pursue a nonintegrated training pathway, and 2 others would pursue a different specialty altogether.

Several features common to I- 6 curricula correspond to the overwhelming satisfaction with the I- 6 format. Among the respondents surveyed, $81 \%$ were satisfied with their operative experience thus far (Table 3), because $23 \%, 31 \%$, and $46 \%$ of respondents anticipated performing more than 200 cardiac cases by the end of their fourth, fifth, and sixth years, respectively (Figure 1). Lower general thoracic case numbers were noted; $19 \%, 17 \%$, and $17 \%$ of respondents anticipated performing more than 200 cases over the same years. Dedicated time for didactic teaching and conferences appears to be emphasized in I- 6 curricula, because $96 \%$ of respondents verified this educational component, with $87 \%$ of these residents receiving at least 2 hours of teaching per week. Technical 
TABLE 1. General survey information and demographics

\begin{tabular}{lc}
\hline \multicolumn{2}{c}{ Parameter } \\
\hline ACGME-approved I-6 residency programs in 2013 & 25 \\
ACGME-approved I-6 residency programs with & 21 \\
$\quad$ currently enrolled residents & \\
ACGME-approved I-6 residency programs with & \\
$\quad$ survey respondents & 57 \\
No. of survey respondents & $69 \%$ \\
Survey response rate & \\
Survey respondent demographics & \\
$\quad$ Men & $77 \%(\mathrm{n}=44)$ \\
Women & $23 \%(\mathrm{n}=13)$ \\
Median age (y) & 29 \\
PGY distribution & \\
$\quad$ PGY1 & $37 \%(\mathrm{n}=20)$ \\
PGY2 & $19 \%(\mathrm{n}=10)$ \\
PGY3 & $21 \%(\mathrm{n}=13)$ \\
PGY4 & $14 \%(\mathrm{n}=8)$ \\
PGY5 & $5 \%(\mathrm{n}=4)$ \\
PGY6 & $4 \%(\mathrm{n}=2)$ \\
\hline
\end{tabular}

Survey respondent breakdown by program

\begin{tabular}{lc}
\hline \multicolumn{1}{c}{ Integrated program } & Respondents (n) \\
\hline Cleveland Clinic & 2 \\
Emory University & 3 \\
Indiana University & 2 \\
Medical College of Wisconsin & 3 \\
Medical University of South Carolina & 2 \\
Mount Sinai School of Medicine & 4 \\
New York Presbyterian (Columbia) & DNP \\
Northwestern University & 3 \\
Stanford University & 7 \\
University of California Davis & 0 \\
University of Kentucky & 1 \\
University of Maryland & 3 \\
University of Michigan & 2 \\
University of North Carolina at Chapel Hill & 5 \\
University of Pennsylvania & 4 \\
University of Pittsburg Medical Center & 2 \\
University of Rochester & 2 \\
University of Southern California & 3 \\
University of Texas Health Science Center & 5 \\
$\quad$ at San Antonio & \\
University of Virginia & 1 \\
University of Washington & 3
\end{tabular}

Data are presented as percentage of 57 total respondents (\%) or absolute number (n). $A C G M E$, Accreditation Council for Graduate Medical Education; DNP, did not participate; $I-6$, integrated 6-year; $P G Y$, postgraduate year.

training by faculty or advanced fellows outside of the operating room and simulation systems for cardiothoracic surgical or interventional procedures were available to $72 \%$ and $91 \%$ of respondents, respectively. Finally, faculty mentorship during training was acknowledged by $95 \%$ of respondents.

Despite overall satisfaction with the I-6 format, more operative exposure $(58 \%)$, more didactic teaching $(42 \%)$, and
TABLE 2. Motivations for pursuing a career in cardiothoracic surgery and integrated 6-year training

\begin{tabular}{lr}
\hline Exposure to cardiothoracic surgery in medical school \\
Cardiothoracic surgical rotations \\
$\quad$ Time on rotations \\
$\quad$ None & $93 \%$ \\
1 mo & $7 \%$ \\
2 mo & $12 \%$ \\
3 mo & $18 \%$ \\
$\quad \geq 4$ mo & $32 \%$ \\
$\quad$ Active participation in cases & $32 \%$ \\
$\quad 0-10$ cases & \\
$\quad 11-50$ cases & $16 \%$ \\
$\quad>50$ cases & $37 \%$ \\
Factors influencing decision to pursue cardiothoracic surgical career \\
Prior clinical exposure & $47 \%$ \\
Interest in cardiovascular disease & $98 \%$ \\
Faculty mentorship & $96 \%$ \\
Patient characteristics & $89 \%$ \\
New technologies & $79 \%$ \\
Prior research in the field & $72 \%$ \\
Income potential/future earnings & $63 \%$ \\
I-6 format itself & $44 \%$ \\
Factors influencing decision to pursue I-6 training & $46 \%$ \\
Focused curriculum & \\
Integration of cardiovascular medicine & $100 \%$ \\
Less general surgical training & $82 \%$ \\
Shorter time in training & $81 \%$ \\
Integration of pulmonary medicine & $74 \%$ \\
Research opportunities & $60 \%$ \\
Earlier income potential & $53 \%$ \\
\hline Data are presented as percentage of 57 total respondents (\%). I-6, Integrated $6-y e a r$ \\
\end{tabular}

more elective rotations $(37 \%)$ were programmatic changes most frequently identified by the surveyed residents (Table 3 ).

\section{Future Career Plans and Outlook}

Among the respondents, career plans skewed toward adult cardiac $(67 \%)$, followed by pediatric cardiac $(24 \%)$ and general thoracic $(9 \%)$ surgery, with $93 \%$ anticipating an academic career (Table 4). Clinical or basic research activity was anticipated in $68 \%$ and $23 \%$ of the respondents, respectively, and many aspired to become a Division or Section Chief $(61 \%)$. Of note, $78 \%$ of respondents devoted some time to conducting research during their training thus far, mainly in the domain of cardiovascular disease $(68 \%)$. Although only $23 \%$ of respondents were afforded dedicated research time during their training, $63 \%$ of those surveyed engaged in research for more than 6 months.

Despite the higher perceived salary ranges relative to academic practice, only $26 \%$ of respondents anticipated pursuing a private practice career. Most I-6 residents $(88 \%)$ planned to pursue additional subspecialty fellowship training (eg, aortic, heart failure, minimally invasive, congenital) after completing their programs. All surveyed residents believe that formal training in cardiac 
TABLE 3. Satisfaction with and prevalence of integrated 6-year program curricular elements

\begin{tabular}{lr}
\hline \multicolumn{1}{c}{ Satisfaction } \\
\hline Satisfaction with I-6 program & \\
Very satisfied & $57 \%$ \\
Satisfied & $38 \%$ \\
Neutral & $5 \%$ \\
Dissatisfied & $0 \%$ \\
Very dissatisfied & $0 \%$ \\
If given another chance... & \\
$\quad$ Would still pursue I-6 training & $93 \%$ \\
$\quad$ Would pursue traditional cardiothoracic training program & $4 \%$ \\
$\quad$ Select another field & $4 \%$ \\
Satisfaction with overall operative experience & $81 \%$ \\
\hline
\end{tabular}

\begin{tabular}{|c|c|}
\hline Prevalence of I-6 curricular elements & \\
\hline \multicolumn{2}{|l|}{ Participation in research } \\
\hline Engaged in any research during training? & $71 \%$ \\
\hline Dedicated research time? & $23 \%$ \\
\hline \multicolumn{2}{|l|}{ Time devoted to conducting research? } \\
\hline$<6$ mo & $38 \%$ \\
\hline $6-12 \mathrm{mo}$ & $23 \%$ \\
\hline$>12 \mathrm{mo}$ & $40 \%$ \\
\hline Cardiovascular research? & $68 \%$ \\
\hline \multicolumn{2}{|l|}{ Training } \\
\hline Didactic training? & $96 \%$ \\
\hline $1 \mathrm{~h} / \mathrm{wk}$ & $13 \%$ \\
\hline $2 \mathrm{~h} / \mathrm{wk}$ & $58 \%$ \\
\hline$>3 \mathrm{~h} / \mathrm{wk}$ & $29 \%$ \\
\hline$>6 \mathrm{~h} / \mathrm{wk}$ & $0 \%$ \\
\hline Technical training with faculty/fellow? & $72 \%$ \\
\hline Exposure to surgical simulation? & $95 \%$ \\
\hline Clinical proficiency appropriate at current training level? & $67 \%$ \\
\hline \multicolumn{2}{|l|}{ Suggested programmatic improvements? } \\
\hline More operative experience & $58 \%$ \\
\hline More didactic teaching & $42 \%$ \\
\hline More elective rotations & $37 \%$ \\
\hline More nonoperative rotations & $19 \%$ \\
\hline More intensive care unit rotations & $19 \%$ \\
\hline More general surgical rotations & $7 \%$ \\
\hline Formal training in cardiac catheterization & $100 \%$ \\
\hline
\end{tabular}

catheterization should be incorporated into the I- 6 curricula, with $91 \%$ foreseeing that cardiothoracic surgeons will eventually perform catheter-based procedures.

With respect to career opportunities after graduating, $49 \%$ of respondents were not particularly concerned about future employment and thought that I-6 program graduates had a distinct advantage over traditionally trained graduates in the job market. In fact, $65 \%$ foresaw an increase in placement opportunities on graduation.

\section{DISCUSSION}

Over the 6 years that have passed since the adoption of the I-6 format at Stanford in 2007, a picture of the characteristics and consequences of this nascent training paradigm is just now coming into sharper focus. The overarching goals of this new training strategy were (1) to develop a more focused and relevant curriculum to produce cardiothoracic surgeons better equipped to practice modern cardiothoracic surgery and (2) to attract a greater number of highly qualified trainees to the field.

Although the realization of these goals has yet to be definitively established, early indicators are promising. Since the initial adoption of the I-6 format, there has been a steady growth in the number of ACGME-approved I-6 programs in the United States, with 14 programs offered in the 2011-2012 match ${ }^{10}$ and 23 programs in the 2013-2014 match. Acceptance into these programs has been highly competitive, with more than 100 applications received by most programs for 1 or 2 positions each year. Furthermore, there is evidence indicating that the qualifications of the I- 6 applicants are generally superior to those of applicants to traditional cardiothoracic surgical training programs. Chikwe and colleagues ${ }^{11}$ at Mt Sinai Medical Center in New York and Gasparri and colleagues ${ }^{12}$ at the Medical College of Wisconsin reported higher numbers of peer-reviewed publications and higher US Medical Licensing Examination scores among applicants for their I-6 program compared with their traditional program applicants. Although these parameters are not firmly established indicators for success as a surgical resident, they reflect positively on the assimilation and retention of curriculum and interest in research.

Conversely, some concerns about the I-6 format have arisen, including a perception that these programs are cardiac focused, uncertainty as to the willingness of cardiothoracic surgical faculty to train junior residents (particularly in the operating room), heterogeneity of the curriculum among different programs, vulnerability to midcourse resident attrition, and concerns related to the lack of maturity and clinical acumen otherwise obtained in traditional 5-year general surgical training programs. ${ }^{10,12}$ Indeed, the "direct-entry" integrated cardiac surgical residencies established in Canada in the early 1990s faced and continue to grapple with challenges similar to those currently faced with the US programs. The direct-entry general thoracic surgical programs did not succeed because graduates from these programs were only certified to perform general thoracic surgery; residents preferred to also complete general surgical training to provide them with more employment opportunities. In Canada, a significant proportion of general thoracic cases are performed by general surgeons, putting direct-entry thoracic graduates at a competitive disadvantage. Although these circumstances do not exist in the United States to the same degree, maintaining balance in US integrated program curricula and emphasizing rotational experiences relevant to general thoracic surgery would seem to be prudent. 


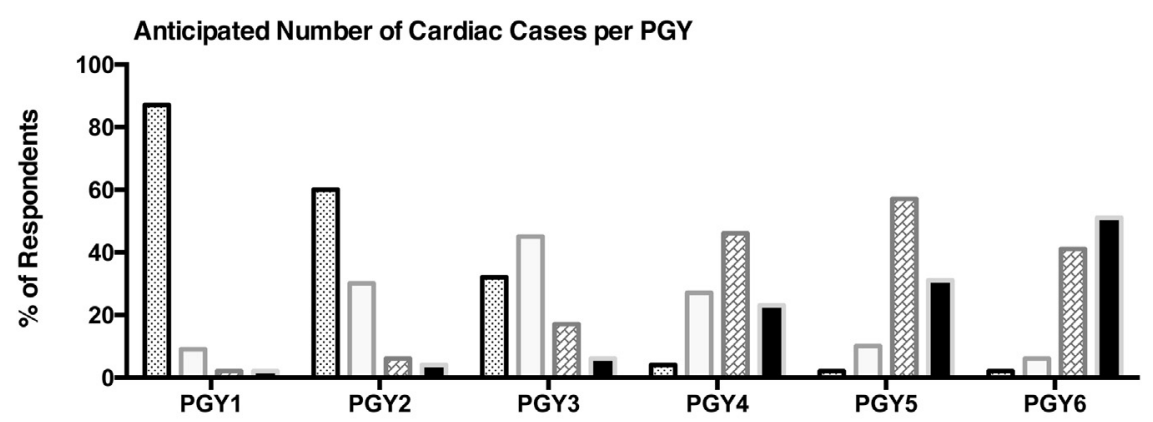

Anticipated Number of General Thoracic Cases per PGY

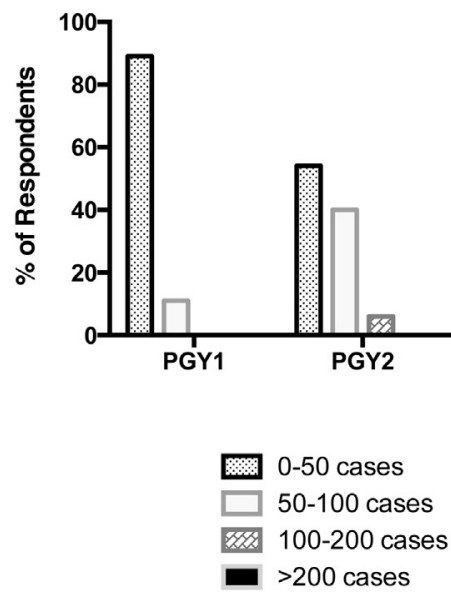

FIGURE 1. The I-6 residents' anticipated cardiac and general thoracic case volumes per PGY. Data represented as percentages of 57 total respondents. $P G Y$, Postgraduate year.

In acknowledgment of the perceived advantages and disadvantages of the I- 6 format, the success or failure of this format will largely depend on the satisfaction of its participants, including their impressions of the quality of training provided and their desired and achieved career paths. In an effort to provide a snapshot of these objectives at an early stage of the format's evolution, we conducted a nationwide survey of I-6 residents and interpreted them according to the following domains.

\section{Resident Characteristics}

The survey data confirm the critical importance of substantial and sustained experiential exposure to cardiothoracic surgery and faculty mentorship in attracting medical students to the field of cardiothoracic surgery. It seems that the focused curriculum and format of I-6 programs were significant draws to the field in many respondents. We were particularly impressed that $26 \%$ of I-6 resident respondents earned additional advanced degrees (eg, $\mathrm{PhD}, \mathrm{MPH}, \mathrm{MSc}$ ) and that $70 \%$ actively engaged in cardiovascular research before entering the residency. These characteristics, coupled with an overwhelming $95 \%$ of respondents intending to pursue an academic career, indicate an academic phenotype among
I-6 applicants. Whether this characteristic endures over the ensuing 6 years of training remains to be seen.

\section{Satisfaction With the Integrated 6-Year Format}

We observed that $95 \%$ of surveyed I-6 residents were "satisfied" or "highly satisfied" with their programs and that $93 \%$ would not have chosen an alternative pathway if given the chance. Several features stood out in detailing this overall satisfaction. As expected, the focused curriculum, exposure to cardiovascular and pulmonary medicine, and reduced time spent on general surgical rotations were seen as the most important advantages of the I- 6 format among the respondents. Of note, $81 \%$ of respondents were satisfied with their operative experience thus far, with substantial proportions of the PGY4, PGY5, and PGY6 residents anticipating performing more than 200 cardiac cases by the end of their respective years of training. Substantial dedicated time for didactic teaching seems to be uniform among I- 6 programs, with the majority of trainees receiving 2 or more hours of didactic teaching per week and faculty- or fellow-mediated technical training outside of the operating room. Training with simulator systems in cardiothoracic surgery or interventional cardiology appears to be de rigueur among I-6 programs. 
TABLE 4. Future career plans and outlook

\begin{tabular}{lr}
\hline Anticipated practice after graduation? & \\
Adult cardiac surgery & $67 \%$ \\
Congenital cardiac surgery & $24 \%$ \\
General thoracic surgery & $9 \%$ \\
Academic practice & $95 \%$ \\
Private practice & $26 \%$ \\
Plans to pursue additional training after graduation & \\
$\quad$ (eg, fellowship)? & \\
Congenital cardiac surgery & $26 \%$ \\
Cardiopulmonary transplantation & $17 \%$ \\
Minimally-invasive cardiothoracic surgery & $15 \%$ \\
Thoracic aortic surgery & $15 \%$ \\
Mechanical circulatory support & $13 \%$ \\
Critical care & $2 \%$ \\
Unsure & $11 \%$ \\
Career outlook concerns? & \\
Not at all & $9 \%$ \\
Slightly & $40 \%$ \\
Moderately & $32 \%$ \\
Strongly & $14 \%$ \\
Very strongly & $5 \%$ \\
Career placement opportunities in future... & \\
Will increase & $65 \%$ \\
Will decrease & $28 \%$ \\
Will remain the same & $7 \%$ \\
I-6 training provides distinct advantages in job market? & $49 \%$ \\
\hline Data are presented as percentage of 57 total respondents (\%). I-6, Integrated $6-y e a r$. \\
\hline
\end{tabular}

Finally, the fact that faculty mentorship was acknowledged by $95 \%$ of respondents should be reassuring to those that harbor concerns about faculty dedication to I-6 residents.

The respondents identified several areas of improvement. More operative exposure (58\%), more didactic teaching $(42 \%)$, and more elective rotations $(37 \%)$ were the programmatic changes most frequently mentioned. Indeed, the integration of cardiovascular and pulmonary medical rotations was viewed as a valuable feature of their respective programs.

Our observation that most I-6 residents planned to pursue additional subspecialty fellowship training (eg, aortic, heart failure, minimally invasive, congenital) after graduating was not surprising, because this has also been observed with traditional cardiothoracic training programs. It is also not surprising that most surveyed residents believe that formal training in cardiac catheterization should be incorporated into I-6 curricula, with almost all anticipating that cardiothoracic surgeons will eventually perform catheter-based procedures. Certainly, an increasing number of cardiothoracic surgical residents are being introduced to catheter-based procedures, including thoracic endovascular aortic repair and transcatheter aortic valve replacement.

\section{Cardiac Bias in Integrated 6-Year Programs?}

Although the general thoracic operative case numbers were lower across all PGY categories, this did not translate into dissatisfaction with the programs among the respondents. Nevertheless, the fact that only $9 \%$ of surveyed residents envisioned a career in general thoracic surgery compared with careers in adult $(67 \%)$ and pediatric $(24 \%)$ cardiac surgery is noteworthy. These observations are relevant to the perception that many I-6 programs are considered to be cardiac focused, despite conflicting evidence. Tchantchaleishvili and colleagues ${ }^{13}$ reported less interest in a thoracic surgery track among I-6 program applicants, but hypothesized that individuals interested in general thoracic surgery were self-selecting out of the I-6 match to train in general thoracic surgery programs and pathways. Ward and colleagues ${ }^{10}$ found that I-6 residents spend less time on general thoracic rotations than dedicated 3 -year general thoracic pathways, but actually more than in traditional cardiothoracic training programs. Whether or not I-6 programs are truly "cardiac-centric," it appears that they face competition from other training pathways in general thoracic surgery.

\section{Career Outlook}

The degree to which career plans of I-6 residents were skewed toward adult and pediatric cardiac surgery was surprising, particularly with renewed interest in general thoracic surgery among applicants to traditional cardiothoracic surgical training programs. There are several potential explanations. First, it is possible that medical students are not afforded as much clinical exposure to general thoracic surgery as general surgical residents. Second, the perception that I-6 programs are more cardiac focused may dissuade applicants interested in pursuing a career in general thoracic surgery, particularly with alternatives including 2- and 3-year "thoracic-track" programs. Finally, it is not unreasonable to consider that medical students applying to I-6 programs may naively and superficially view cardiac surgery as more "glamorous" than general thoracic surgery.

As mentioned earlier, our survey indicates an early preference toward academic careers, with $91 \%$ of respondents anticipating conducting research in their careers and a majority aspiring to academic leadership. However, given the limited numbers of I-6 programs affording dedicated research time to their trainees, it is questionable whether the interest, aptitude, and skills required to develop into a successful academician can be consistently developed in I-6 residents, particularly with the escalating challenges in acquiring extramural research funding.

We were struck by the general optimism among I-6 residents in terms of career placement opportunities, with approximately half of those surveyed not particularly concerned about finding a suitable position on graduation and a clear majority foreseeing an increase in future job opportunities. In fact, approximately half of our respondents thought that graduating from an I-6 program would 
constitute a competitive advantage over graduates from traditional programs. It is worth mentioning that, among our respondents, the level of financial compensation did not appear to be a major consideration in making career choices; this finding is even more significant given that residents' perceptions of opening salary levels were below those of recently published estimates. Only $26 \%$ of respondents anticipated pursuing a private practice career, despite a higher perceived income compared with academic careers.

\section{Study Limitations}

Although we achieved an excellent $69 \%$ response rate and robust sample size given the relatively small numbers of I-6 residents and programs, we recognize several significant limitations of our survey. First, given that the first I-6 residents graduated earlier this year, our survey sample was heavily skewed toward trainees in their first 3 PGYs. Although we presume that the initial motivations for pursuing careers in cardiothoracic surgery and applying to I-6 programs would be definitive regardless of the point in training, the impressions gleaned from our survey may not hold steady as the junior residents progress through their training. Second, it is possible that respondents were biased toward residents who were pleased with their I-6 programs. Because we could not achieve a $100 \%$ response rate, eliminating the chances of such behavioral bias was not possible. Third, except for operative case volumes, we did not stratify the responses according to PGY because the differences in sample sizes between the different strata were too significant to make meaningful comparisons between individual years. Consequently, we were not able to ascertain variance in opinions among residents in the different stages in training. Third, the paucity of PGY6 residents to survey limits our ability to gauge the success of the I-6 format in developing confident, well-rounded cardiothoracic surgical residents who attain early career success and satisfaction. It would be important for future surveys to determine whether the strong preference toward academic careers reflected in this survey is actually realized among I-6 graduates. Finally, although we do not think that our modest monetary incentive to participate in our detailed survey was a confounding factor, it is conceivable that this skewed our sampling in some unforeseen way. We thought that increasing the likelihood of a robust survey response in the interest of arriving at truly representative conclusions outweighed any theoretic bias.

Many of the responses prompted additional questions that we would have liked to have posed. For example, given that an overwhelming majority of surveyed residents enjoyed faculty mentorship, it would have been useful to determine whether this was facilitated by the 6-year format versus traditional 2- or 3-year pathways. Furthermore, given the surprisingly low proportion of I-6 residents intending to pursue a career in general thoracic surgery, additional questions designed to ascertain why would have been illuminating.

\section{CONCLUSIONS}

This survey takes an important snapshot of the nascent I-6 format, providing insight in determining (1) whether this format is achieving its overarching mission of attracting more highly qualified trainees to the field and producing surgeons well versed in contemporary cardiothoracic surgery, (2) whether current residents are satisfied with their quality of training, and (3) what features of currently heterogeneous I-6 residency curricula are most effective in achieving the desired goals. We confirmed that early mentorship and intense clinical exposure are critical in attracting applicants to the field and that the purported advantages of the I-6 format are currently holding true among I-6 residents, with a high level of satisfaction. Furthermore, these early data indicate that the I-6 format is a "work-in-progress" but holds significant promise in attracting, retaining, and producing highly qualified cardiothoracic surgeons.

The authors thank all I-6 residents who responded to our survey and Suzanne Giannotti, who provided us assistance in coordinating this study. The William Glenn Research Fund from the Yale University School of Medicine Department of Surgery funded this study.

\section{References}

1. Reddy VS, Calhoon JH. Cardiothoracic surgical education: the ideal platform for tomorrow's surgeon. Tex Heart Inst J. 2010;37:656-7.

2. Cooke DT, Kerendi F, Mettler BA, Boffa DJ, Mehall JR, Merrill WH, et al. Update on cardiothoracic surgery resident job opportunities. Ann Thorac Surg. 2010;89:1853-9

3. Schlitzkus LL, Schenarts KD, Schenarts PJ. Is your residency program ready for Generation Y? J Surg Educ. 2010;67:108-11.

4. Salazar JD, Ermis P, Laudito A, Lee R, Wheatley GH 3rd, Paul S, et al. Cardiothoracic surgery resident education: update on resident recruitment and job placement. Ann Thorac Surg. 2006;82:1160-5.

5. Vaporciyan AA, Reed CE, Erikson C, Dill MJ, Carpenter AJ, Guleserian KJ, et al. Factors affecting interest in cardiothoracic surgery: survey of North American general surgery residents. J Thorac Cardiovasc Surg. 2009;137:1054-62.

6. Grover A, Gorman K, Dall TM, Jonas R, Lytle B, Shemin R, et al. Shortage of cardiothoracic surgeons is likely by 2020. Circulation. 2009;120:488-94.

7. Merrill WH. Preparing the next generation of residents to care for patients with cardiothoracic disease. Tex Heart Inst J. 2012;39:878-9.

8. Rusch VW, Calhoon JH, Allen MS, Baumgartner W. The American Board of Thoracic Surgery: update. J Thorac Cardiovasc Surg. 2012;143:519-21.

9. Pousatis SM, Marshall MB. Trends in applications for thoracic fellowship in comparison with other subspecialties. Ann Thorac Surg. 2014;97:624-33.

10. Ward ST, Smith D, Andrei AC, Hicks GL Jr, Shemin RJ, Calhoon JH, et al Comparison of cardiothoracic training curricula: integrated six-year versus traditional programs. Ann Thorac Surg. 2013;95:2051-6.

11. Chikwe J, Brewer Z, Goldstone AB, Adams DH. Integrated thoracic residency program applicants: the best and the brightest? Ann Thorac Surg. 2011;92: 1586-91.

12. Gasparri MG, Tisol WB, Masroor S. Impact of a six-year integrated thoracic surgery training program at the Medical College of Wisconsin. Ann Thorac Surg. 2012;93:592-7.

13. Tchantchaleishvili V, Barrus B, Knight PA, Jones CE, Watson TJ, Hicks GL. Six-year integrated cardiothoracic surgery residency applicants: characteristics, expectations, and concerns. J Thorac Cardiovasc Surg. 2013;146:753-8. 